\title{
Molecular Association of Tetramethylurea and Chlorobenzene Molecules in Microwave Frequency Range
}

\author{
Vimal Sharma ${ }^{\mathrm{a}}$ and Nagesh Thakur ${ }^{\mathrm{b}}$ \\ a Dept. of Physics, NIT, Hamirpur, (H. P.) India \\ b Dept. of Physics, H. P. University, Shimla, (H. P.) India \\ Reprint requests to V. S.; E-mail: vimal16sharma@yahoo.co.in
}

Z. Naturforsch. 65a, 854 - 858 (2010); received August 17, 2009 / revised December 19, 2009

\begin{abstract}
The dielectric constant $\varepsilon^{\prime}$ and dielectric loss $\varepsilon^{\prime \prime}$ of the binary mixtures of tetramethylurea (TMU) and chlorobenzene (CB) have been calculated at $9.883 \mathrm{GHz}$ by using standard standing microwave techniques. Gopalakrishna's single frequency concentration variation method has been used to calculate dipole moment $\mu$ and dielectric relaxation time $\tau$ for different mole fractions of TMU in the binary mixture at different temperatures of $25{ }^{\circ} \mathrm{C}, 30{ }^{\circ} \mathrm{C}, 35^{\circ} \mathrm{C}$, and $40{ }^{\circ} \mathrm{C}$. The variation of dielectric relaxation time with the mole fraction of TMU in the whole concentration range of the binary mixtures was found to be non-monotonic. The solute-solute and solute-solvent type of molecular associations may be proposed based upon above observations. Using Eyring rate equations the energy parameters $\Delta H, \Delta F$, and $\Delta S$ for the dielectric relaxation process and the viscous flow process have been calculated at the given temperatures. It is found from the comparison of energy parameters that, just like the viscous flow process, the dielectric relaxation process can also be treated as a rate process.
\end{abstract}

Key words: Tetramethylurea (TMU); Chlorobenzene (CB); Binary Mixture;

Microwave Absorption; Dielectric Relaxation.

\section{Introduction}

The study of dielectric relaxation of polar liquids in non-polar solvents subjected to electromagnetic waves in microwave frequency region have thrown much light on the size, shape, and structure of the polar molecules. The dispersion region of polar molecules lies in the microwave region of the electromagnetic spectrum and therefore these waves are capable to respond to even weak molecular interactions [1-5]. In the present work the dielectric relaxation properties of the binary mixtures of TMU and chlorobenzene in bezene solutions at $9.883 \mathrm{GHz}$ frequency and $25{ }^{\circ} \mathrm{C}$, $30{ }^{\circ} \mathrm{C}, 35{ }^{\circ} \mathrm{C}$, and $40{ }^{\circ} \mathrm{C}$ temperatures have been studied. Chlorobenzene is an important non-aqueous solvent with the dielectric constant $\varepsilon^{\prime}=5.649$ and the dipole moment $\mu=1.69 \mathrm{D}$ [6]. It is used for manufacturing several important products, such as DDT, nitrochlorobenzene, aniline, and silicone resins [7]. Tetramethylurea is a non-aqueous solvent with dielectric constant $\varepsilon^{\prime}=23.45 \pm 0.06$ and dipole moment $\mu=3.37 \mathrm{D}$ [8]. The combination of high dipole moment, moderate dielectric constant, low viscosity, and low specific conductance makes TMU a useful sol- vent for studying the behaviour of electrolytes in solutions [8]. Benzene is a non-aqueous, non-polar solvent, and has a $\pi$-delocalized electron cloud in the ring. It provides an active medium for solute-solvent association.

It is possible to prepare binary mixtures of tetramethylurea and chlorobenzene having dielectric constants and dipole moment values in between those of TMU and CB. Tetramethylurea and chlorobenzene have quite different static dielectric constants and dipole moments. This motivated the authors to perform experimental studies concerning the dielectric relaxation process in binary mixtures of TMU and $\mathrm{CB}$ in order to understand the molecular association in the whole concentration range of TMU in the binary mixture.

Measurements of dielectric constant $\varepsilon^{\prime}$, dielectric loss $\varepsilon^{\prime \prime}$, dielectric relaxation time $\tau$, and dipole moment $\mu$ have been made for binary mixtures with different mole fractions of TMU $(0.0,0.3,0.5,0.7,1.0)$ at different temperatures $\left(25^{\circ} \mathrm{C}, 30^{\circ} \mathrm{C}, 35^{\circ} \mathrm{C}\right.$, and $\left.40^{\circ} \mathrm{C}\right)$ in the non-polar solvent benzene. The plots of dielectric relaxation time versus mole fraction of TMU in the binary mixtures at different temperatures propose 
solute-solute associations. The energy parameters $\Delta H$, $\Delta F, \Delta S$ have been calculated for the 0.5 -mole fraction of TMU in the binary mixture. It was found that the dielectric relaxation process is a rate process like the viscous flow process.

\section{Experimental Details}

Tetramethylurea (Fluka) was dried over anhydrous $\mathrm{BaO}$ powder for 48 hours and then it was distilled through a long vertical fractionating column. The middle fraction was used with in a week to avoid decomposition. Benzene GR (Grade) from E. Merck Ltd., Mumbai (India), was dried by refluxing over freshly cut sodium metal and then distilled through a long vertical fractionating column. Chlorobenzene from central Drug House (p) Ltd., New Delhi (India), was distilled through a long vertical fractionating column. The middle fraction of each solution was collected for use. The X-band microwave bench was used to measure the wavelength of microwaves in a waveguide $\left(\lambda_{\mathrm{g}}\right)$, in a dielectric $\left(\lambda_{\mathrm{d}}\right)$, in free space $\left(\lambda_{0}\right)$, and the cutoff wavelength $\lambda_{\mathrm{c}}$. The voltage standing wave ratio (VSWR) was also measured. The dielectric constant and the dielectric loss of the dilute solutions of the binary mixtures of TMU and chlorobenzene in benzene at various temperatures $\left(25^{\circ} \mathrm{C}, 30^{\circ} \mathrm{C}, 35^{\circ} \mathrm{C}\right.$, and $\left.40^{\circ} \mathrm{C}\right)$ were calculated following the microwave absorption technique of Heston et al. [9]. Circulating the thermostated water around the dielectric cell, the solution was kept at the desired constant temperature. The viscosities and densities of the solutions were measured by an ubbelohde viscometer and a sealable type of pycnometer, respectively.

\section{Results and Discussion}

The values of $\varepsilon^{\prime}$ (dielectric constant) and $\varepsilon^{\prime \prime}$ (dielectric loss) for the binary mixtures of different mole fractions of TMU in the benzene solution have been calculated using the microwave absorption technique of Heston et al. [9].

The accuracy in measurements of $\varepsilon^{\prime}$ and $\varepsilon^{\prime \prime}$ was $1 \%$ and $3 \%$, respectively. The equations used to calculate $\varepsilon^{\prime}$ and $\varepsilon^{\prime \prime}$ are

$$
\begin{aligned}
& \varepsilon^{\prime}=\left(\frac{\lambda_{0}}{\lambda_{\mathrm{c}}}\right)^{2}+\left(\frac{\lambda_{0}}{\lambda_{\mathrm{d}}}\right)^{2}, \\
& \varepsilon^{\prime \prime}=\frac{2}{\pi}\left(\frac{\lambda_{0}}{\lambda_{\mathrm{d}}}\right)^{2} \frac{\lambda_{\mathrm{g}}}{\lambda_{\mathrm{d}}} \frac{\mathrm{d} \rho}{\mathrm{d} n} .
\end{aligned}
$$

Here $\lambda_{0}, \lambda_{\mathrm{c}}, \lambda_{\mathrm{g}}$, and $\lambda_{\mathrm{d}}$ are the wavelength of microwaves in free space, the cut off wavelength, the waveguide wavelength, and the wavelength in the waveguide filled with the solution, respectively, and $\rho$ is the inverse of the voltage standing wave ratio. $(\mathrm{d} \rho / \mathrm{d} n)$ is the slope of the curve of $\rho$ versus $n$, where $n$ is an integer $(n=1,2,3, \ldots)$, such that $\left(n \lambda_{\mathrm{d}} / 2\right)$ represents the length of the dielectric filled waveguide.

The single frequency and concentration variation method of Gopala Krishna [10] was used to calculate the relaxation time $\tau$ and dipole moment $\mu$ of the molecular entities:

$$
\begin{aligned}
X & =\frac{\varepsilon^{\prime 2}+\varepsilon^{\prime 2}+\varepsilon^{\prime}-2}{\left(\varepsilon^{\prime}+2\right)^{2}+\varepsilon^{\prime \prime 2}}, \\
Y & =\frac{3 \varepsilon^{\prime \prime}}{\left(\varepsilon^{\prime}+2\right)^{2}+\varepsilon^{\prime 2}}, \\
\tau & =\frac{\lambda_{0}}{2 \pi c}\left(\frac{\mathrm{d} Y}{\mathrm{~d} X},\right) \\
\mu^{2} & =\frac{9 k T M}{4 \pi N \mathrm{~d}}\left[1+\left(\frac{\mathrm{d} Y}{\mathrm{~d} X}\right)^{2}\right] \frac{\mathrm{d} X}{\mathrm{~d} W} .
\end{aligned}
$$

Here $X$ and $Y$ are intermediate parameters which depend upon $\varepsilon^{\prime}$ and $\varepsilon^{\prime \prime} . W$ is the weight fraction of the binary mixture (TMU $+\mathrm{CB}$ ) in benzene.

The energy parameters $\Delta H_{\varepsilon}, \Delta F_{\varepsilon}, \Delta S_{\varepsilon}$ for the dielectric relaxation process of the binary mixtures in benzene have been calculated and compared with the corresponding energy parameters for a viscous flow process $\Delta H_{\eta}, \Delta F_{\eta}, \Delta S_{\eta}$, using the Eyring rate equations [11]. Following equations have been used to calculate the energy parameters:

$$
\begin{aligned}
& \tau=\frac{h}{k T} \exp \left[\frac{\Delta F_{\varepsilon}}{R T}\right], \\
& \Delta F_{\varepsilon}=\Delta H_{\varepsilon}-T \Delta S_{\varepsilon}, \\
& \eta=\left(\frac{h N}{V}\right) \exp \left[\frac{\Delta F_{\eta}}{R T}\right], \\
& \Delta F_{\eta}=\Delta H_{\eta}-T \Delta S_{\eta} .
\end{aligned}
$$

The values of the dielectric relaxation time $\tau$ and the dipole moment $\mu$ for binary mixtures of different mole fractions of TMU $(0.0,0.3,0.5,0.7,1.0)$ at different temperatures $\left(25{ }^{\circ} \mathrm{C}, 30{ }^{\circ} \mathrm{C}, 35^{\circ} \mathrm{C}\right.$, and $\left.40{ }^{\circ} \mathrm{C}\right)$ have been tabulated (Table 1). Table 2 represents the values of $\Delta H_{\varepsilon}, \Delta F_{\varepsilon}, \Delta S_{\varepsilon}$ and $\Delta H_{\eta}, \Delta F_{\eta}, \Delta S_{\eta}$. 
Table 1. Dielectric relaxation time $\tau$ and dipole moment $\mu$ for different mole fractions of (TMU $+\mathrm{CB})$ mixtures in benzene solution at different temperatures.

\begin{tabular}{lccc}
\hline$T$ & mole fraction of & $\tau$ & $\mu$ \\
${ }^{\circ} \mathrm{C}$ & TMU in binary mixture & $10^{-12} \mathrm{~s}$ & $\mathrm{D}$ \\
\hline 25 & 0.00 & 4.88 & $\mu_{\mathrm{TMU}}=3.16$ \\
& 0.30 & 5.84 & \\
& 0.50 & 8.58 & \\
& 0.70 & 7.31 & \\
& 1.00 & 7.01 & $\mu_{\mathrm{CB}}=1.63$ \\
& 0.00 & 4.67 & $\mu_{\mathrm{TMU}}=3.36$ \\
30 & 0.30 & 5.56 & \\
& 0.50 & 8.20 & \\
& 0.70 & 6.85 & \\
& 1.00 & 6.25 & $\mu_{\mathrm{CB}}=1.63$ \\
& 0.00 & 4.46 & $\mu_{\mathrm{TMU}}=3.37$ \\
& 0.30 & 5.21 & \\
& 0.50 & 7.78 & \\
& 0.70 & 6.35 & \\
& 1.00 & 5.58 & $\mu_{\mathrm{CB}}=1.66$ \\
& 0.00 & 4.26 & $\mu_{\mathrm{TMU}}=3.46$ \\
& 0.30 & 4.88 & \\
& 0.50 & 7.34 & \\
& 0.70 & 5.77 & \\
& 1.00 & 4.91 & $\mu_{\mathrm{CB}}=1.70$ \\
\hline
\end{tabular}

\subsection{Dielectric Constant and Dielectric Loss}

The plots of $\varepsilon^{\prime}$ and $\varepsilon^{\prime \prime}$ versus the weight fraction $W$ of the solute in benzene for all binary mixtures were found to be linear. This shows that the molecular entities retain their configuration, shape, and size in the studied concentration range of the binary mixtures in the benzene solutions and ensures the applicability of Debye's theory [12] and Gopala Krishna's method. Debye's theory is related to dielectric dispersion in polar complexes and according to Debye, the liquid polar molecule subjected to an electric field may be considered as a rotating sphere surrounded by an environment of finite viscosity.

\subsection{Relaxation Time}

The relaxation time for pure TMU is found to be more than that of pure $\mathrm{CB}$ at all temperatures. The relaxation time increases with the size of the molecule and may be discussed in terms of the molecular shape and solvent microscopic viscosity. This shows that the size of a TMU molecule is more than that of a chlorobenzene molecule. The decrease in the relaxation time with rise in temperature can be explained on the basis of the Debye theory [12]. Thermal energy of the system increases with rise in temperature and decreases the relaxation time of the molecular entities.
Table 2. Enthalpies of activation $\left(\Delta \mathrm{H}_{\varepsilon}, \Delta \mathrm{H}_{\eta}\right.$ in $\mathrm{kJ}$ mole $\left.{ }^{-1}\right)$, free energies of activation $\left(\Delta \mathrm{F}_{\varepsilon}, \Delta \mathrm{F}_{\eta}\right.$ in $\left.\mathrm{kJ} \mathrm{mole}^{-1}\right)$, and entropies of activation $\left(\Delta \mathrm{S}_{\varepsilon}, \Delta \mathrm{S}_{\eta}\right.$ in $\left.\mathrm{kJ} \mathrm{mole}^{-1} \mathrm{~K}^{-1}\right)$ for (TMU $+\mathrm{CB}$ ) mixture containing 50 -mole $\%$ of TMU in benzene solutions.

\begin{tabular}{ccccccc}
\hline $\begin{array}{c}\text { Temperature } \\
{ }^{\circ} \mathrm{C}\end{array}$ & $\begin{array}{c}\Delta \mathrm{H}_{\varepsilon} \\
\pm 0.17\end{array}$ & $\begin{array}{c}\Delta \mathrm{F}_{\varepsilon} \\
\pm 014\end{array}$ & $\begin{array}{c}\Delta \mathrm{S}_{\varepsilon} \\
\pm 0.31\end{array}$ & $\Delta \mathrm{H}_{\eta}$ & $\Delta \mathrm{F}_{\eta}$ & $\Delta \mathrm{S}_{\eta}$ \\
\hline 25 & 5.643 & 9.863 & -14.15 & 11.02 & 12.20 & -3.96 \\
30 & 5.643 & 9.957 & -14.23 & 11.02 & 12.23 & -3.99 \\
35 & 5.643 & 10.03 & -14.23 & 11.02 & 12.25 & -3.99 \\
40 & 5.643 & 10.08 & -14.17 & 11.02 & 12.32 & -4.15 \\
\hline
\end{tabular}

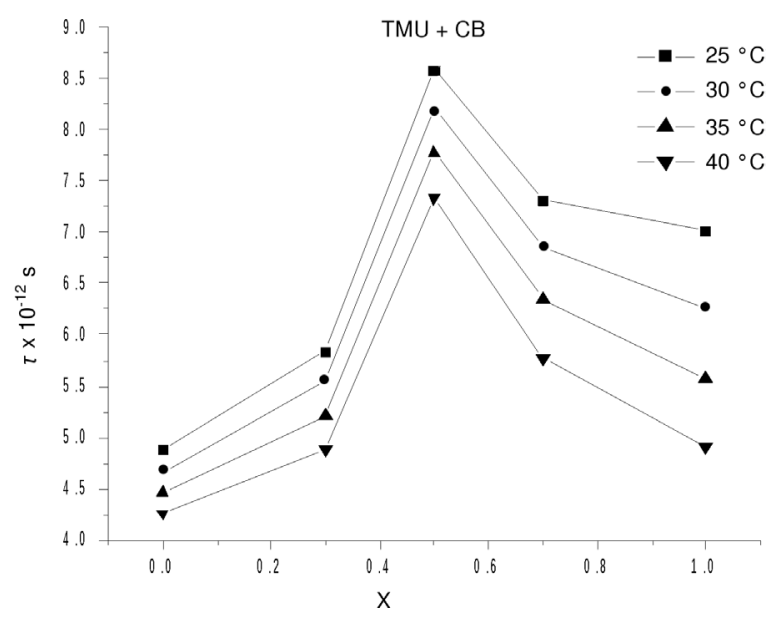

Fig. 1. Variation of relaxation time with mole fraction $X$ of TMU in the binary mixture $(\mathrm{TMU}+\mathrm{CB})$ in benzene solution at different temperatures.

At higher temperature, due to a larger number of collisions, the rate of energy loss increases, hence, reorientation of molecules becomes faster.

The non-monotonic variation of the dielectric relaxation time with the mole fraction of TMU in the binary mixtures at all temperatures was observed (Fig. 1). The monotonic variation of the relaxation time from its value corresponding to one single constituent to the value which corresponds to the pure other constituent, with varying mole fraction in the whole concentration range may be taken as the absence of any solute-solute association in the mixture [13]. On the other hand, the non-monotonic variation of the relaxation time with the mole fraction is interpreted as a possible solutesolute molecular association in the binary mixture [14]. In the present study, the non-monotonic variation of the relaxation time with the change in mole fraction of TMU in the binary mixture was observed. The relaxation time increases sharply with the increase in mole fraction from 0.0 to 0.3 . The peak is formed at 0.5 mole fraction of TMU in the binary solution and 


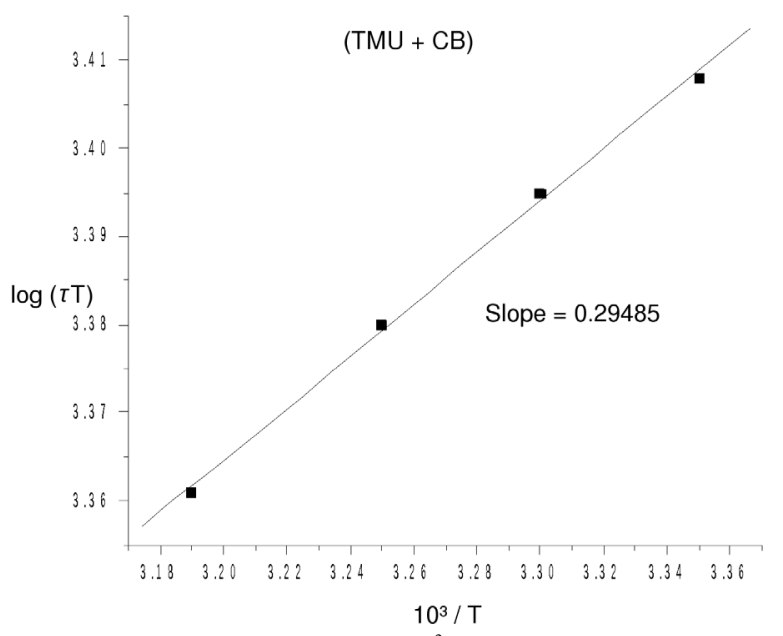

Fig. 2. Plot of $\log (\tau T)$ versus $\frac{10^{3}}{T}$ for 0.5 mole fraction of TMU in the binary mixture (TMU + CB).

the relaxation time attains a maximum value at this mole fraction. With the further increase in the mole fraction of TMU in the mixture there is a decrease in the relaxation time. It falls towards the value of pure TMU in the benzene solution. This predicts the solutesolute type of molecular association between TMU and CB which may arise because of the dipole-dipole interaction between tetramethylurea and chlorobenzene molecules [15] caused by the presence of fractional negative charge at the site of the oxygen atom of TMU and positive charge on the chlorine atom of chlorobenzene molecule.

\section{Dipole Moment}

The calculated dipole moments of TMU and chlorobenzene were found to be very near to those in the literature. This shows that pure TMU and pure chlorobenzene exist in the monomer form in benzene solution. Dipole moment of the molecular entities depends on the size, structure, and charge separation in the molecular entities. The variation of the dipole moment with rise in temperature shows a solutesolvent association while no change in dipole moment with temperature shows the absence of solute-solvent

[1] N. Thakur and D. R. Sharma, Indian J. Pure Appl. Phys. 38, 328 (2000)

[2] A. D. Vyas and V. A. Rana, Indian J. Pure Appl. Phys. 40, 69 (2002).

[3] V.S. Rangra and D. R. Sharma, Indian J. Phys. B 78, 111 (2004) association. The small change in the dipole moment with temperature may be due to the stretching of bond moments and the change in bond angles. This change may also be due to the breaking of solute-solvent associations with rise in temperature.

\section{Energy Parameters}

The plot of $\log (\tau T)$ versus $\frac{10^{3}}{T}$ for 0.5 mole fraction of TMU in the binary mixtures (Fig. 2) is found to be linear which indicates that the dielectric relaxation process can be treated as a rate process. Energy parameters $\Delta H_{\varepsilon}, \Delta F_{\varepsilon}, \Delta S_{\varepsilon}$ for the dielectric relaxation process and the viscous flow process $\left(\Delta H_{\eta}, \Delta F_{\eta}, \Delta S_{\eta}\right)$ have been calculated and compared. It is found that the free energy of activation of the dielectric relaxation process is less than that of the viscous flow process. This may be explained with the argument that the dielectric relaxation process involves the rotation of molecular entities, whereas in the flow process the rotational as well as the translational motions of the molecules are involved. The enthalpy of activation depends on the local environment of the molecules. The enthalpy of activation of the dielectric relaxation process was found to be different from that of a viscous flow process. This difference shows that the dielectric relaxation process comprises different types of bonding and breaking of the bonding to different extents.

According to Branin and Smyth [16], a negative value of the entropy of activation indicates that a comparatively smaller number of configurations is possible in an activated state, which is more ordered in comparison to the normal state. This again indicates the presence of a cooperative orientation of the molecules by the steric forces or by strong dipole-dipole interaction with the dipole being better aligned in an activated state. However, the positive value of the activation entropy indicates that the activated state is more disordered. In the present case it is observed that the change in entropy of the dielectric relaxation process is negative indicating that the environment of the system is cooperative like that of the activated viscous flow state.

[4] A. Chaudhari, S. Ahire, and S. C. Mehrotra, J. Mol. Liq. 94, 17 (2001).

[5] G. D. Rewar and D. Bhatnagar, Indian J. Pure Appl. Phys. 40, 430 (2002).

[6] N.E. Hill, W.E. Vaughan, A.H. Price, and M. Davies, Dielectric Properties and Molecu- 
lar Behaviour, Van Nostrand-Reinhold, London 1969, p. 2.

[7] E. Kilner and D. M. Samuel, Applied Organic Chemistry, MacDonald and Evans Ltd., London 1960, p. 244.

[8] J. J. Lagowski, The Chemistry of Non-aqueous Solvents, Academic Press, New York, San Francisco, London 1976, p. 111.

[9] W. M. Heston, Jr., A. D. Franklin, E. L. Hennely, and C. P. Smyth, J. Am. Chem. Soc. 72, 3443 (1950).

[10] K. V. Gopala Krishna, Trans. Faraday Soc. 33, 767 (1957).
[11] H. Eyring, S. Glasstone, and K. J. Laidler, Theory of Rate Process, Mc Grow-Hill, New York 1941, p. 541.

[12] P. Debye, Polar molecules, Chemical Catalog Co, New York 1929.

[13] V. Sharma and N. Thakur, Z. Naturforsch. 63a, 93 (2008).

[14] S. Kumar, D. R. Sharma, N. Thakur, N.S. Negi, and V. S. Rangra, Indian J. Pure Appl. Phys. 44, 264 (2006).

[15] N. Thakur and D. R. Sharma, Indian J. Pure Appl. Phys. 41, 806 (2003).

[16] F. H. Branin, Jr. and C. P. Smyth, J. Chem. Phys. 20, 1121 (1952). 